\title{
Numerical investigation on the elastic modulus of rubber-like materials by a rigid ball indentation technique
}

\author{
Budi Setiyana ${ }^{1, *}$, Sugiyanto ${ }^{1}, J$. Jamari ${ }^{1}, M$. Khafidh $^{2}$ \\ ${ }^{1}$ Laboratory for Engineering Design and Tribology, University of Diponegoro, 50275 Semarang, \\ Indonesia \\ ${ }^{2}$ Laboratory for Surface Technology and Tribology, University of Twente, 7500 AE Enschede, The \\ Netherlands
}

\begin{abstract}
The indentation technique has been practically proven to be useful in determining mechanical properties of materials, such as hardness and elastic modulus for rubber-like materials (elastomers). However, tensile test method is often conducted because of obtaining the mechanical strength in addition to the elastic modulus of the elastomer. In this paper, a numerical study is proposed to investigate the elastic modulus of the elastomer by applying Finite Element Analysis (FEA). With the availability of Strain Energy Function (SEF) data from the material testing, the investigation is carried out by indentation technique for Natural Rubber (NR) and Styrene Butadiene Rubber (SBR). On the rubber surface, a rigid ball indenter is pressed under specified indentation force and the contact depth resulted is observed. Based on the ASTM (American Society for Testing and Materials) formulation, the elastic modulus from the indentation technique can be estimated. In general, results show that the elastic modulus obtained from the indentation technique agree with the tensile test results. Thus, the proposed numerical method is validly applied in determining the elastic modulus.
\end{abstract}

\section{Introduction}

The indentation technique has been proven useful in estimating the mechanical properties of materials, especially the hardness of materials. In rubber and soft material technology, the indentation test has been known to be a measure of an elastic modulus [1,2] that is a main mechanical properties related to "hardness" of the material. In addition to shore durometer test which is frequently used to measure the rubber hardness, the indentation test was also issued by ASTM [3]. This indentation technique obtains the hardness of rubber by the difference of penetration depth of a specified dimension of ball indenter.

* Corresponding author: bsetiyana@yahoo.com 
An indentation analysis for obtaining the hardness by a spherical and a conical indenter was performed experimentally, analytically, and numerically [4,5]. Results showed that the analysis by using low order of elasticity theory agree with the case of a small depth of indentation of a blunt conical indenter or a large spherical radius. However, for the large depth of penetration, it requires the numerical method that is based on Strain Energy Function (SEF) than the elasticity theory. The Strain Energy Function is associated with the stress-strain relationship which is developed from the model that conform to tension experiments up to a certain level of straining [1].

In rubber abrasion, the periodic pattern on the abraded rubber surface was formed [6]. Experimentally, the interesting result was stated that length of the pattern spacing formed was depended on hardness or elastic modulus of the rubber. High hardness of the rubber forms short pattern spacing and vice versa [7-8]. Therefore, the rubber hardness plays an important role in the rubber abrasion.

American Society for Testing and Materials (ASTM) issued the method to estimate the hardness of rubber by using indentation technique. According to this method, formulation to obtain the rubber hardness and the elastic modulus are presented. It depends on the depth of indentation (penetration), ball indenter dimension and indenter force. The elastic modulus of rubber can be determined by a tensile test method but it is not simple to conduct such test compared to the indentation test. However, tensile test method is often conducted because of obtaining the mechanical strength in addition to the elastic modulus. On the other side, it is difficult to find accurately the elastic modulus of the elastomer by theoretical formulation due to its complex material behaviour.

In this paper, a numerical study is proposed to investigate of the elastic modulus of the elastomer by indentation technique. The investigation is carried out by Finite Element Analysis (FEA) for unfilled Natural Rubber (NR-0), unfilled Styrene Butadiene Rubber (SBR-0) and filled Styrene Butadiene Rubber (SBR-25). It requires the Strain Energy Function (SEF) data from the testing results. With FE simulation, a rigid ball indenter with 0.5 tip radius is pressed and the contact depth resulted are observed. Based on the ASTM formulation, the elastic modulus from the indentation technique can be estimated.

\section{Methods}

The finite element analysis of the present work was performed using a commercial finite element software package, ABAQUS 6.11 [9] with some built-in strain energy function model for a hyperelastic material. A rigid ball indenter was pressed on an elastomer surface. The $0.5 \mathrm{~mm}$ tip radius were used. The unfilled Natural Rubber (NR), unfilled Styrene Butadiene Rubber (SBR-0) and filled Styrene Butadiene Rubber with carbon black (SBR-25). Mooney-Rivlin model of Strain Energy Function (SEF) was used for NR and SBR-0, meanwhile Yeoh model was used for SBR-25. The SEF data were adopted from Liang's experiment [10].

Figure 1(a) shows a schematic illustration of the rigid ball indentation of an elastomeric surface in two dimensional view, where $\mathrm{F}$ is the indenter load, $\mathrm{r}$ is the tip radius and $\delta$ is the depth of contact, while the thickness of the elastomeric material is perpendicular to this figure. Boundary conditions of the contact system are depicted in this figure, that the rubber can freely move in vertical direction. The elastomer material was modeled as three dimension with a $10 \mathrm{~mm}$ height, $20 \mathrm{~mm}$ width and $10 \mathrm{~mm}$ thickness. 


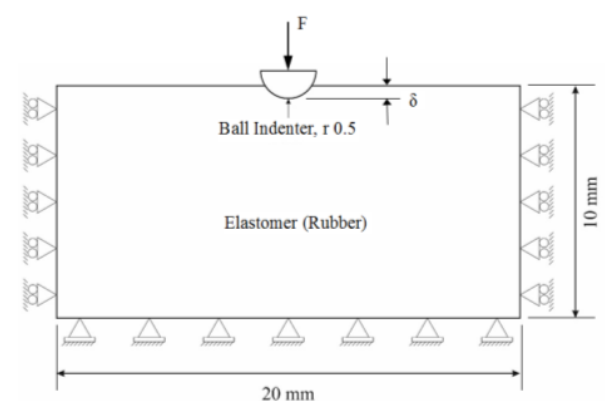

(a)

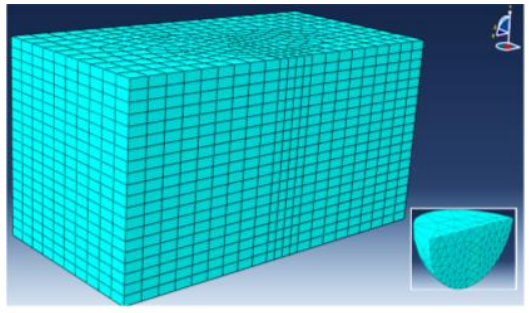

(b)

Fig. 1. (a) Schematic 2D section illustration of the indentation model; a rigid ball on the rubber surface and (b) the 3D section of generated FEA mesh for rubber and ball indenter (inset).

The generated mesh of FE model of the indentation is depicted in Fig. 1(b) that consists of the rubber and ball indenter meshing (inset) in 3D section. In starting on FE simulation, the rigid ball indenter is pressed with specified force on the top of the rubber surface. The results are presented in the form of the indenter force with respect to the depth of indentation. Based on the ASTM formulation, the elastic modulus can be estimated. Furthermore, the comparison results between the indentation technique and the tensile test method are carried out.

\section{Results and Discussion}

The results of FE simulation in rubber indentation are imaged in Figure 2. By using $1.0 \mathrm{~mm}$ ball indenter diameter, Fig. 2(a) shows the contour of the deformed surface around the ball indenter in 2D section for various depth of indentation i. e. $0.3,0.5$ and $0.7 \mathrm{~mm}$. Meanwhile, the deformed sufaces and von Mises stress distribution in 3D section around the ball indenter are demonstrated in Fig. 2(b). It can be seen the degree of stress intensity around the ball indenter that are represented as specified colour. Regarding to this figure, the stress intensity is shown in symmetric form and the highest stress are located below the indenter tip.

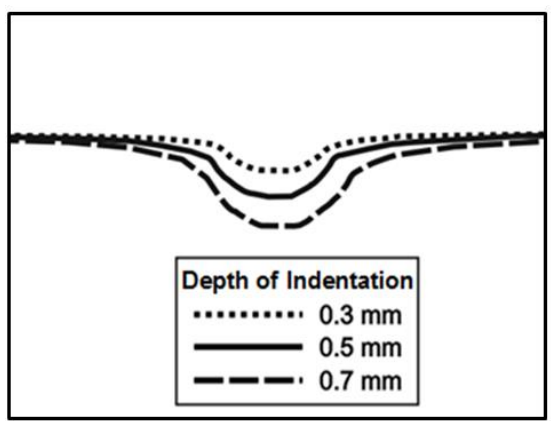

(a)

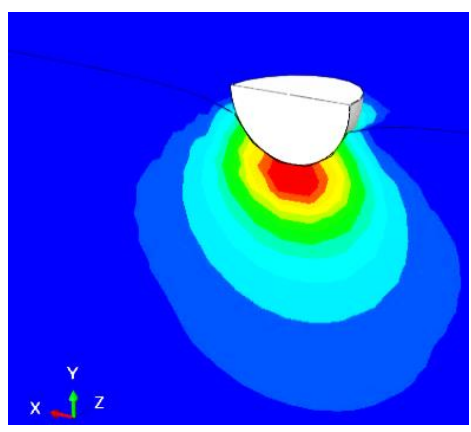

(b)

Fig. 2. The results of FE simulation in rubber indentation (a) The 2D section of the deformed surface contour in various depth of indentation (b) The 3D section of deformation and stress distribution of $0.7 \mathrm{~mm}$ depth. 


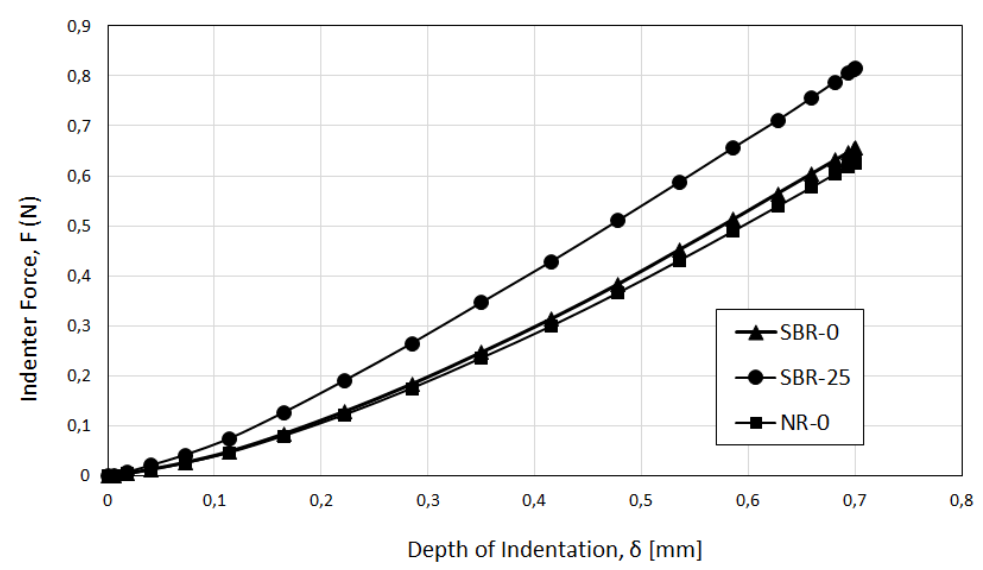

Fig. 3. The relationship between the depth of indentation and the indenter force for various rubber

By performing the numerical method by using FE analysis, the relationship between the depth of indentation $\delta$ and the indentor force $F$ is plotted in Fig. 3. The depth of indentation is given up to $0.7 \mathrm{~mm}$, thus the reaction or indeter force is identified. At the same depth of indentation, material filled Styrene Butadiene Rubber (SBR-25) need higher indenter force than unfilled rubber i. e. SBR-0 and NR-0. Meanwhile, there is slightly different of the indenter force between SBR-0 and NR-0. Regarding to these results, it reflects that the SBR-25 has higher stiffness than SBR-0 as well as NR-0. In general, rubber with high stiffness is also belong to the high hardness and elastic modulus.

Based on the indentation technique, ASTM proposes a formulation to obtain the elastic modulus $\mathrm{E}$ of the rubber as following [3],

$$
E=\frac{F}{1.9 r^{2}(\delta / r)^{1.35}}
$$

Where $\mathrm{E}$ is elastic modulus (MPa), $\mathrm{F}$ is indenter force $(\mathrm{N}), \mathrm{r}$ is radius of ball indenter $(\mathrm{mm})$ and $\delta$ is the depth of penetration or indentation $(\mathrm{mm})$. Based on the data and results from FE simulation above, the elastic modulus of the rubber can be obtained by applying the Eq.1. The resulted elastic modulus with respect to the depth of indentation is plotted in Fig. 4. It is shown that the elastic modulus has high value for small depth (around 0 to 0.05 $\mathrm{mm}$ ), however, it has relatively constant value for middle and large depth of indentation. There is slightly difference value of the elastic modulus between SBR-0 and NR-0 that is around 1.7 to $2.0 \mathrm{Mpa}$. On the other hand, material SBR-25 has a high elastic modulus with the value around 2.4 to $2.6 \mathrm{Mpa}$. 


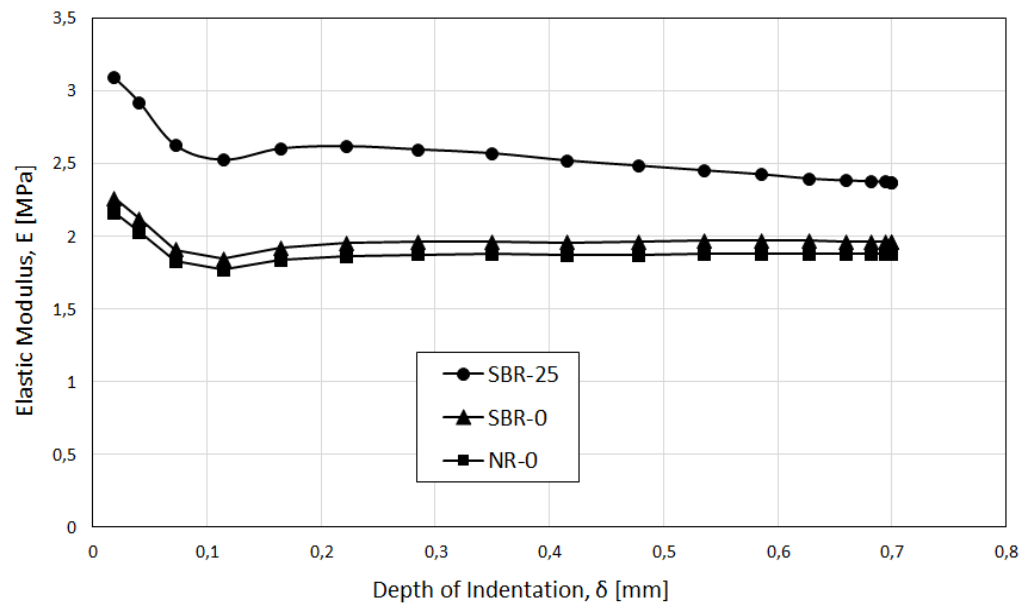

Fig. 4. The elastic modulus of various rubber resulted from indentation technique by means of FE Analysis

The elastic modulus is commonly constructed from tensile test data. In tensile test method, the stress-strain relationship is obtained that is commonly presented as engineering stress $\sigma_{\text {eng }}$ versus stretch ratio $\lambda$, i.e. final length divided by initial length of the rubber specimen. Regarding to the rubber theory [1], the true stress can be obtained by using the Eq. 2. Furthermore, the elastic modulus E can be obtained by means of the Eq. 3 .

$$
\begin{aligned}
\sigma_{\text {true }}=\lambda & \sigma_{\text {eng }} \\
& E=\frac{\sigma_{\text {true }}}{\varepsilon}=\frac{\sigma_{\text {true }}}{\lambda-1}
\end{aligned}
$$

Equation 3 states that the elastic modulus is obtained by dividing the true stress $\sigma_{\text {true }}$ by strain $\varepsilon$. The value of the strain $\varepsilon$ relates to the stretch ratio, that is $\varepsilon=\lambda-1$.

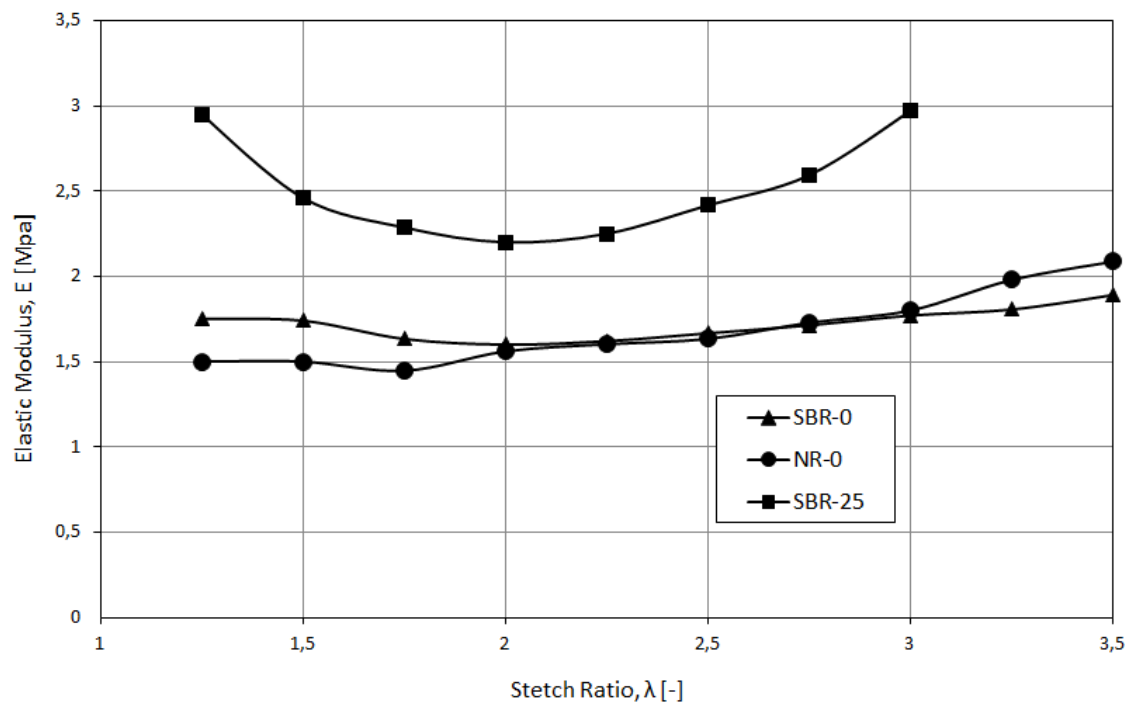

Fig. 5. The elastic modulus of various rubber resulted from the tensile test method 
Figure 5 shows the elastic modulus of the tensile test method. The tensile test data are adopted from Liang's experiment in form as stress-strain relationship [9]. By using Eq. 2 and Eq. 3, the elastic modulus are presented with respect to the stretch ratio. It can be seen that the elastic modulus for SBR-0 and NR-0 are around 1.5 to 2.0, meanwhile, the elastic modulus for SBR-25 is around 2.2 to 2.9. In general, the elastic modulus of the tensile test results is agree with the indentation technique, and thus, the numerical method used in this paper is validly applied.

\section{Conclusion}

In this paper, a numerical study is proposed to investigate of the elastic modulus of the elastomer by indentation technique. By using provided Strain Energy Function (SEF) data from material testing, the investigation is carried out by Finite Element Analysis (FEA) for Natural Rubber (NR) and Styrene Butadiene Rubber (SBR). A rigid ball indenter with 0.5 tip radius is pressed and contact depth resulted is observed. Based on the ASTM formulation, the elastic modulus from the indentation technique is numerically estimated. Regarding to the numerical simulation, the filled rubber (SBR-25) has a higher elastic modulus than unfilled rubber (SBR-0 and NR-0). The elastic modulus of SBR-25 is around 2.4 to 2.6, meanwhile, for unfilled rubber is around 1.7 to 2.0 Mpa. Results show that the elastic modulus resulted from the indentation technique agree with the tensile test results. Thus, the proposed numerical method used is validly applied.

\section{References}

1. A.N. Gent, Engineering with Rubber (Carl Hanser Verlag Press, New York, 1992)

2. M. Czerner, L.S. Fellay, M.P. Suárez, P.M. Frontini, L.A. Fasce, Determination of Elastic Modulus of Gelatin Gels by Indentation Experiments, Procedia Mat. Sci. 8, 287-296 (2015)

3. ASTM Designation D 1415-88, Standard Test Method for Rubber PropertyInternational Hardness (Reapproved 1999)

4. A.E. Giannakopoulos, D.I. Panagiotopoulos, Spherical indentation of incompressible rubber-like materials, Int. J. Sol. and Struct. 55, 1196 (2007)

5. A.E. Giannakopoulos, D.I. Panagiotopoulos, Conical indentation of incompressible rubber-like materials, Int. J. Sol. and Struct. 46, 1436 (2009)

6. Y. Fukahori and H. Yamazaki, Mechanism of rubber abrasion - Part 1: abrasion pattern formation in natural rubber vulcanizate, Wear 171, 195 (1994)

7. Y. Fukahori and H. Yamazaki, Mechanism of rubber abrasion - Part 2: General rule in abrasion pattern formation in rubber-like materials, Wear 178, 109 (1994)

8. B. Setiyana, R. Ismail, J. Jamari and D.J. Schipper, Stick-Slip Behaviour of a Viscoelastic Flat Sliding Along a Rigid Indenter, Tribol. Online 11, 512-518 (2016)

9. ABAQUS 6.11/Standard User's Manual (Dassault Systems Simulia Corp., USA, 2011)

10. H. Liang, Investigating the Mechanism of Elastomer Abrasion ( $\mathrm{PhD}$ Thesis, University of London, 2007) 